\title{
Speed Control of Vibration Micro-motors of a Micro-Robotic Platform
}

\author{
Iliana Spartali* \\ Email: ilianaspartali@gmail.com
}

\author{
Kostas Vlachos* \\ Email: kostaswl@central.ntua.gr
}

\author{
Evangelos Papadopoulos* \\ Email: egpapado@central.ntua.gr
}

\begin{abstract}
In this paper we consider the dynamic modeling and the speed control of vibration micro-motors, under power and sensor hardware constraints due to the centimeter-scale dimensions of the motors. The micro-motors are attached on a centrifugal-force micro-robotic mobile platform. The dynamic model of the low cost motor is presented, and discussed. The experimental procedure for the motors' parameter identification is presented, and the design of a model-based controller aiming at the control of the speed of the motors is analyzed. In addition, the practical implementation of the designed controller is discussed and experimentally evaluated. In order to measure the rotational speed of each centimeterscale vibration motor, a rotary encoder with one count per revolution is designed and constructed. Despite this sensor hardware limitation, the experimental results indicate that the micro-motors were successfully modeled, and that the proposed controller results in the minimization of the steady-state error and in an increase of the motors' bandwidth by a factor of three. Additional experiments showed that the application of the model-based speed controller results in a motion of the micro-robotic platform of higher precision.
\end{abstract}

\section{INTRODUCTION}

Over the past years micromanipulation and microengineering tasks have been attractive to several research groups in the international robotics community. An important goal of the research in this field is the realization of autonomous robotic systems characterized by high motion resolution. This leads to the development of mobile micro-robotic platforms that can be used in micro fabrication or biomedical applications, such as cell manipulation and sperm injection [1], [2]. The key component of miniature mobile micro-robots is their actuation mechanism, because it is directly related to the micron-resolution capabilities, the power autonomy and the compact design [3]. Several micro-actuation techniques have been based on smart materials, such as piezoelectric actuators that employ the stick-slip principle, like those used at MINIMAN and MiCRoN robots [4], [5]. Although piezoelectric actuators are the most favored materials for the required positioning resolution and actuation response for micro positioning, they usually suffer from complex power units that are costly and cumbersome and do not allow for untethered operation [6], [7]. Thus, a significant disadvantage of the above mechanisms despite the benefits they offer, is the lack of autonomy. The novel micro robotic

* All authors are with the Department of Mechanical Engineering, National Technical University of Athens, 15780 Athens, Greece. platform which was presented in [8] and [9], does not have the disadvantages described above because it is based on a very different actuation mechanism. It includes two vibration micro-motors to perform translational and rotational sliding and achieves micrometer positioning accuracy. The same actuation principle has been employed by the Kilobot microrobot presented in [10], used to study collective behaviors in swarming applications.

In this paper we design and implement a model-based speed controller on the vibration micro-motors, under power and hardware constraints, of the micro-robot presented in [8], see Fig. 1. Our goal is to improve the motion resolution and the bandwidth of the micro-robot. Early results on the model-based speed control of the micro-motors were presented in [8]. Compared to the previous work, this paper presents a) an alternative, more efficient model-based control law, which leads to a system's response without overshoot and less required power, b) the implementation of the controller on the micro-robot prototype, under power and hardware constraints, and c) experimental results showing that the controller and the micro-robot, despite the constraints, behaves according to the theoretical analysis.

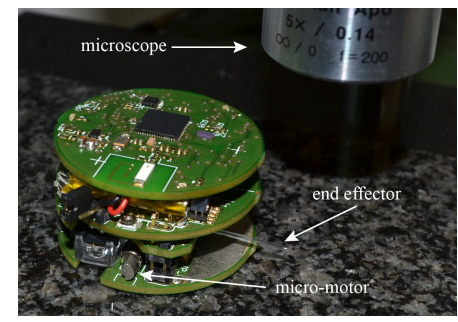

Fig. 1. The micro-robotic platform.

The power constraints are due to the fact that, to operate autonomously, the micro-robotic platform is powered by a battery. In addition, the small dimensions of the micro-motor that is equipped with an eccentric load, made impossible the use of a commercial off-the-self encoder or a tachometer for measuring its rotational speed. To overcome this problem an 1 count per revolution (cpr) encoder is designed and implemented. The motor's mathematical model is formulated, and its parameters were identified. The proposed speed control is based on a model-based scheme using the inverse dynamics of the system. Experiments show that the proposed controller results in the minimization of the steady-state error and in 
an increase of the motor's bandwidth by a factor of three. Additional experiments show that the application of the model-based speed controller results in a more accurate motion of the micro-robotic platform.

\section{Modeling}

\section{A. Dynamics}

The mathematical model of the vibration micro-motor and a number of open loop experiments are presented next. For a detailed description of the innovative actuation principle of the micro-robot one can look in [8], [9]. The actuators of the micro-robot are dc motors with permanent magnet and a shaft coupled with an eccentric mass. The model of the motor and the real motor that was used are shown in Fig. 2a and Fig. 2b respectively. The micro-motor is $8 \mathrm{~mm}$ long (excluding the eccentric mass), and its weight is about $1 g$.

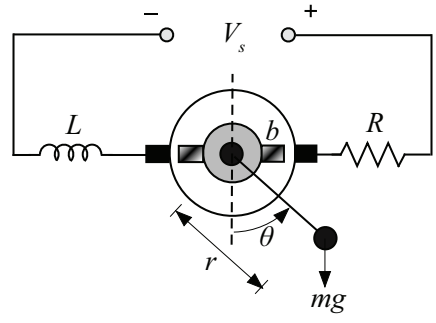

(a)

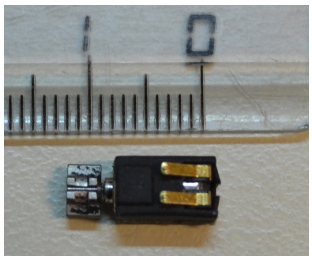

(b)
Fig. 2. (a) Model of the micro-motor. (b) Real micro-motor with eccentric mass.

The dynamics of the micro-motor electrical subsystem is described by the following equation:

$$
L \dot{i_{L}}=V_{s}-K_{T} \dot{\theta}-R i_{L}
$$

where $K_{T}$ is the torque constant, $L$ is the windings inductance, $R$ is the motor's resistance, and $i_{L}$ is the current of the motor. The voltage, $V_{s}$, is the input to the motor, and $\theta$ is the angular position of the eccentric mass. According to the force analysis shown in Fig. 3, the force $m g \sin \theta$ is tangent to the motion, while the force $m g \cos \theta$ cancels out the centripetal force. Hence the torque balance is given by:

$$
J \dot{\omega}=T-c \operatorname{sgn} \omega-b \omega-m g r \sin \theta
$$

where $\omega=\dot{\theta}$ is the rotational speed of the shaft of the motor, $J$ is the inertia of the eccentric mass $m, T$ is the torque, proportional to the current, that drives the motor, $b$ is the viscous friction, $r$ is the distance between the center of the eccentric mass and the shaft of the motor, and the term $c \operatorname{sgn} \omega$ is the Coulomb friction.

From (1) and (2), and if the windings inductance $L$ is neglected, the dynamics of the vibration micro-motor is described by:

$$
\dot{\omega}=\frac{K_{T}}{J R} V_{s}-\frac{b R+K_{T}^{2}}{J R} \omega-\frac{c \operatorname{sgn} \omega}{J}-\frac{m g r \sin \theta}{J}
$$

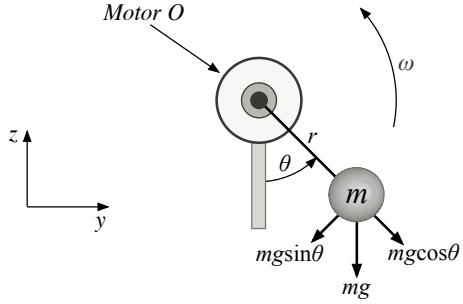

Fig. 3. Force analysis for the eccentric mass.

\section{B. Parameter Identification and Open Loop Experiments}

The identification of the motor's parameters was necessary for the simulation of the dynamic model of the micromotor, and the design of the speed controller. Therefore, a series of experiments were conducted under hardware constraints due to the small size of the low cost motor that was selected. The most important constraint was the lack of an encoder suitable for such a small motor. Consequently, an 1cpr encoder was designed and constructed. Details of the encoder can be found in Section IV.

In order to obtain the torque constant, $K_{T}$, and the viscous friction coefficient, $b$, the motor was led to the steady state, and the parameters were determined using the following equations:

$$
\begin{gathered}
K_{T}=\frac{1}{\omega_{s s}}\left(V_{s}-R i_{L, s s}\right) \\
b=\frac{1}{\omega_{s s}}\left(i_{L, s s} K_{T}-c \operatorname{sgn} \omega_{s s}\right)
\end{gathered}
$$

where $\omega_{s s}$, and $i_{L, s s}$ are the rotational speed, and the current of the motor at the steady-state, respectively. The value of $c$ is given by:

$$
c=i_{L, s} K_{T}
$$

where $i_{L, s}$ is the current required just to start the motor. The mechanical time constant, $\tau_{m e c h}$, is found from the open loop response of the system (3), considering the non-linear terms as a disturbance. Then, the rotor and eccentric mass total inertia, $J$, is given by:

$$
J=\frac{\tau_{\text {mech }}\left(K_{T}^{2}+R b\right)}{R}
$$

The identification results are presented in Table I. These were determined as the average of twenty experimental runs using (4)-(7), or by direct measurements.

TABLE I. MOTOR PARAMETERS

\begin{tabular}{cccc}
\hline $\mathbf{R}[\boldsymbol{\Omega}]$ & $\tau_{\mathbf{m e c h}}[\mathbf{m s}]$ & $\mathbf{K}_{\mathbf{T}}[\mathbf{N m} / \mathbf{A}]$ & $\mathbf{c}[\mathbf{N m}]$ \\
10.7 & 175 & $3.6410^{-4}$ & $1.3410^{-5}$ \\
\hline \hline $\mathbf{b}[\mathbf{N s} / \mathbf{m}]$ & $\mathbf{J}\left[\mathbf{K g m}^{\mathbf{2}}\right]$ & $\mathbf{r}[\mathbf{m m}]$ & $\mathbf{m}[\mathbf{g}]$ \\
$2.9410^{-9}$ & $2.6710^{-9}$ & 1.77 & 0.21 \\
\hline
\end{tabular}

In Fig. 4, open loop simulation and experimental results are presented and compared. In the first pair of plots, the open loop speed response of the simulated and the real micro-motor is shown, while the input voltage, $V_{s}$, is equal to $650 \mathrm{mV}$. In the second pair of plots the input voltage is equal to $850 \mathrm{mV}$. The steady-state value, and the rotational speed at the time equal to the time constant is marked with 
a "star" signs on each plot. We can observe that due to unmodeled dynamics and disturbances to the system, the steady-state open loop rotational speed of the simulation (black line) and the experimental (red line) run have a difference of about $97 \mathrm{rad} / \mathrm{s}$ and $186 \mathrm{rad} / \mathrm{s}$ depending on the input voltage. It is also shown that the time constant of the micro-motor is about $159-183 \mathrm{~ms}$, and that the model successfully predicts it with a small difference of about $23 \mathrm{~ms}$ and $17 \mathrm{~ms}$ depending on the input voltage. In addition, the ripples observed in the steady-state (simulation run) are due to the non-linear term $m g r \sin \theta$. In order to minimize the time constant of the system, and to compensate for the non-linearities and the unmodeled dynamics, a speed controller, described next, is designed and applied.
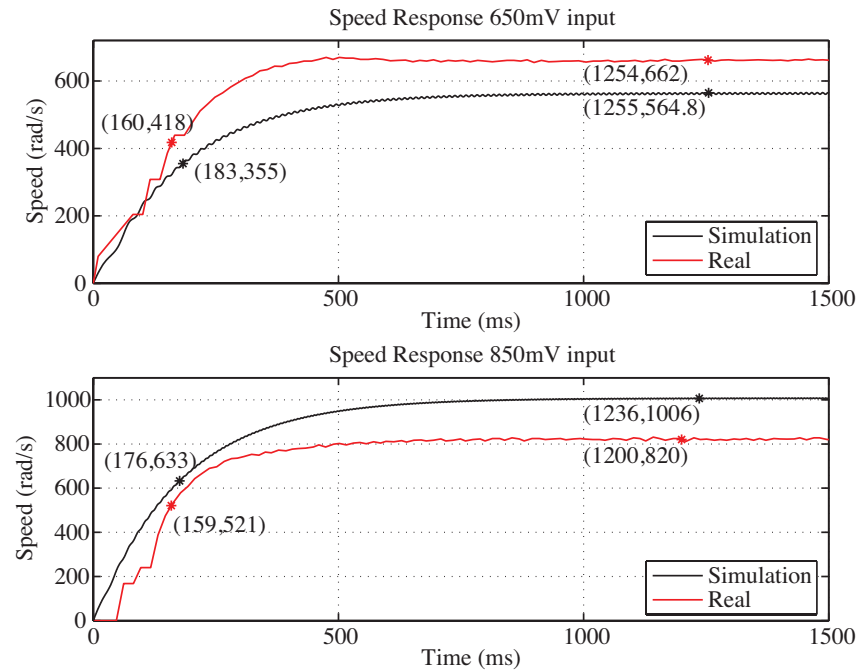

Fig. 4. Open loop speed response for different values of input voltage.

\section{Closed-LoOP CONTROL}

In this section, following a model-based approach, we develop a closed loop speed controller for the micro-motor. The design criteria require to minimize the steady state error, to reduce the time constant of the system, and to minimize the ripples. Additionally, the use of batteries in order to achieve autonomy, impose a power restriction so that the input voltage, $V_{s}$, can not exceed $1.2 \mathrm{~V}$.

From (3), considering there are unknown disturbances $d$ at the system, it follows that:

$$
J \dot{\omega}+\frac{K_{T}^{2}+R b}{R} \omega+c \operatorname{sgn} \omega+m g r \sin \theta=\frac{K_{T}}{R} V_{s}+d
$$

To compensate for the two non-linear terms, we apply the following control voltage:

$$
V_{s}=\frac{K_{T}^{2}+R b}{K_{T}} \omega+\frac{R}{K_{T}} c \operatorname{sgn} \omega+\frac{J R}{K_{T}} u+\frac{R m g r \sin \theta}{K_{T}}
$$

where $u$ is an auxiliary feedback input, which includes proportional, and integral action, and therefore can take care of constant or slow-changing disturbances. Replacing (9) in (8), the system dynamics is written as,

$$
\dot{\omega}=u+\frac{d}{J}
$$

Next, we continue the analysis applying two PI-control laws.

\section{A. PI-Control law A}

The first auxiliary control law is described by the following equation, and a blog diagram of the corresponding closed-loop system is shown in Fig. 5,

$$
u=K_{p} e+K_{I} \int e \mathrm{~d} t
$$

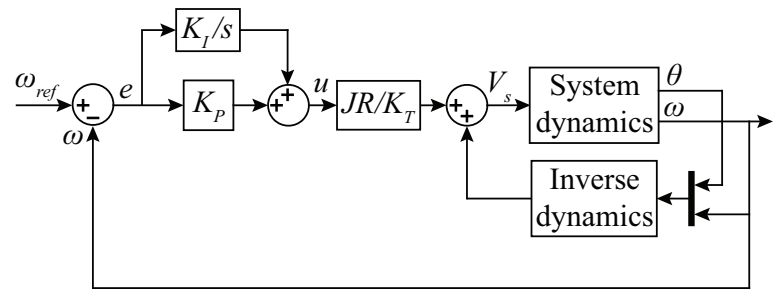

Fig. 5. Closed-loop system with PI-Control law A.

where $e=\omega_{\text {ref }}-\omega$ is the difference between the reference (desired) value of the rotational speed and the measured rotational speed of the micro-motor. Combining (10) and (11), and differentiating under the assumption that the disturbances, $d$, are constant or change very slowly, it follows that:

$$
\ddot{e}+K_{p} \dot{e}+K_{I} e=0
$$

which shows that the error is driven to zero asymptotically. The simulated speed response, for a desired value of $700 \mathrm{rad} / \mathrm{s}$, of the open and closed-loop system is shown in Fig. 6. Although the reference rotational speed is achieved $(698 \mathrm{rad} / \mathrm{s})$, a big overshoot appears at the beginning of the response. This is due to the closed-loop zero introduced by the controller, and due to the fact that the control law requires an input voltage that saturates because of the limited available power, see the lower plot in Fig. 6. Moreover, comparing the open, and closed-loop responses, we see that the settling time of the system is decreased from $692 \mathrm{~ms}$ to $337 \mathrm{~ms}$, i.e a reduction of $51 \%$, and that the modelbased portion of the controller has eliminated the ripples of the speed response. Nevertheless, in order to eliminate the overshoot that appears in the closed-loop response, we design a second control law described next.
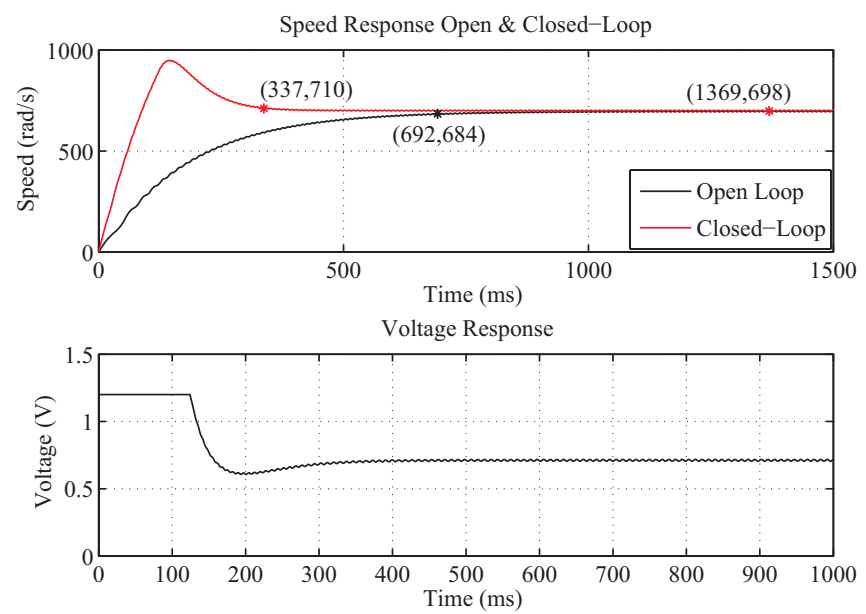

Fig. 6. Simulation results for auxiliary PI-Control law A. 


\section{B. PI-Control law B}

The second auxiliary control law is described by the following equation, and a blog diagram of the corresponding closed-loop system is shown in Fig. 7,

$$
u=-K_{p} \omega+K_{I} \int\left(\omega_{r e f}-\omega\right) \mathrm{d} t
$$

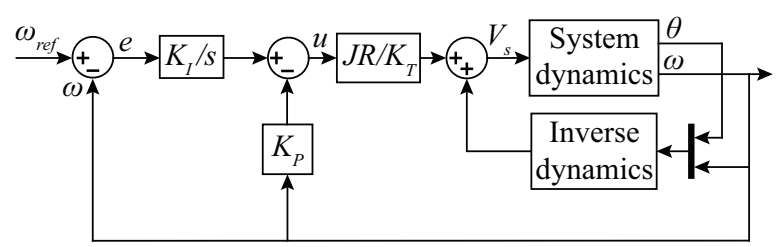

Fig. 7. Closed-loop system with PI-Control B.

Combining (10) and (13), and differentiating under the assumption that the disturbances, $d$, are constant or change very slowly, it follows that:

$$
\ddot{\omega}+K_{p} \dot{\omega}+K_{I} \omega=K_{I} \omega_{r e f}
$$

The system (14) corresponds to a linear second-order system, allowing the selection of the control gains. Transforming to frequency domain, the transfer function is:

$$
G(s)=\frac{\Omega(s)}{\Omega_{r e f}(s)}=\frac{K_{I}}{s^{2}+K_{p} s+K_{I}}
$$

which shows that the error to a step input is driven to zero asymptotically. To avoid oscillations, the poles of (14) are placed at the same point on the left real axis of the complex plane, thus $\zeta=1$. Consequently, the settling time $t_{s}=$ $6 / \omega_{n}$, and:

$$
\begin{aligned}
& K_{I}=\omega_{n}{ }^{2}=36 / t_{s}^{2} \\
& K_{p}=2 \omega_{n}=12 / t_{s}
\end{aligned}
$$

where $\omega_{n}$ is the natural frequency of the system. The desired settling time was chosen taking under consideration that there is a voltage limit $(1.2 \mathrm{~V})$ due to the use of batteries. Thus, we choose a desired settling time of about $243 \mathrm{~ms}$, and using (16), the gains are calculated as $K_{I}=605$ and $K_{p}=49$. The speed response of the open and closed-loop system, for a desired speed $700 \mathrm{rad} / \mathrm{s}$, is shown in Fig. 8.

We can observe that there is no steady-state error, that there is no overshoot, that the ripples are eliminated, and that the settling time of the system has decreased from $709 \mathrm{~ms}$ to $238 \mathrm{~ms}$, i.e a reduction of $65 \%$. In addition, the second diagram of Fig. 8 shows that the required closed-loop input voltage to the micro-motor is under $1.2 \mathrm{~V}$, and there is no saturation which is in accordance with the requirements.

\section{PRACTICAL ImPlementation}

Each vibration micro-motor used as actuator in the micro-robotic platform, is $8 \mathrm{~mm}$ long (excluding the eccentric mass), and its weight is about $1 g$. Consequently, the small dimensions of the motor made impossible the use of a commercial off-the-self encoder or a tachometer in order to measure its rotational speed. Instead, a $1 \mathrm{cpr}$ encoder is designed and implemented. It consists of a low-cost opto-reflective sensor, and the needed electronics. The used

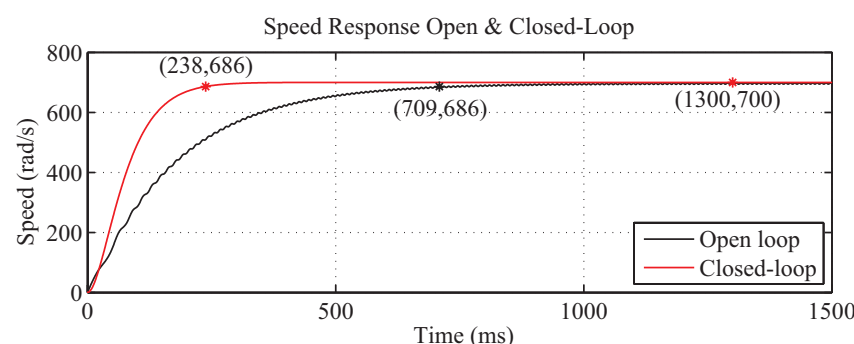

Voltage Response Closed-Loop

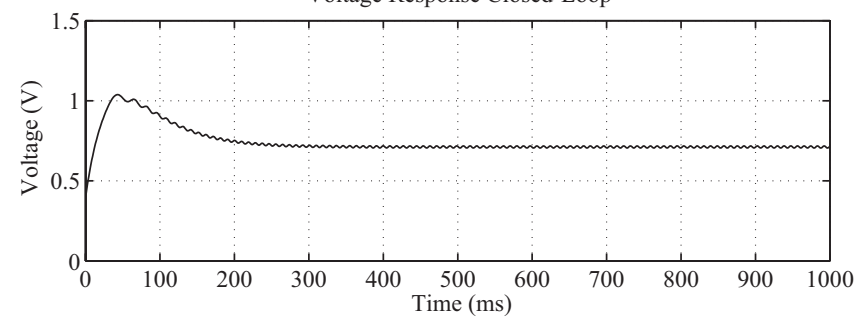

Fig. 8. Simulation results for auxiliary PI-Control law B.

opto-reflective sensor is the QRE-1113 Miniature Reflective Object Sensor by Fairchild Semiconductor, and consists of an infrared led and a phototransistor that does not require contact surface sensing. A sensor is attached on the side of each micro-motor, so that it outputs a pulse when the eccentric mass pass in front of the sensor. Thus the period, $T$, of the pulse signal is measurable and the rotational speed, $\omega$, of the micro-robot is estimated using the equation $\omega=2 \pi / T$. A schematic representation of the principle of operation, and the practical implementation on the microrobotic platform, are shown in Fig. 9.

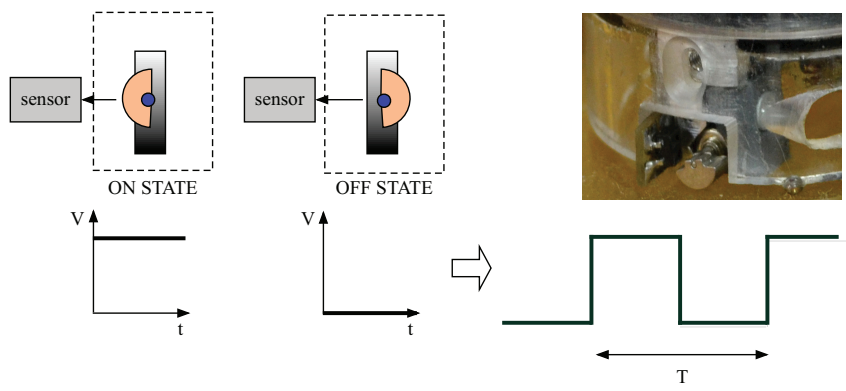

Fig. 9. The sensor placed at a small distance from the motor captures the pulse period.

For implementing the controller, the Microchip PIC18F2431 microcontroller was used. With the aid of real time programming, using the timers of the micro controller, the signals from the sensors are obtained, and transferred via RS232 to a computer for monitoring. The setup for each motor is depicted in Fig. 10. An H-bridge drives each micro-motor, using hard switching. Pulsed Width Modulation was applied at two different channels that are connected to the two halves of the bridge, in order to have bidirectional motion. The voltage, applied by the on-board battery, is $4.9 \mathrm{~V}$ for the microcontroller, and $2.4 \mathrm{~V}$ for the H-bridges of the two motors. As mentioned before, the latter value imposes a significant constraint for the controller since the maximum voltage that can be applied on each motor can not exceed $1.2 \mathrm{~V}$. A $10 \mathrm{Mhz}$ 
quartz crystal was used as a clock oscillator, which allows for adequately accurate PWM values to control the motor voltage. A $100 \%$ PWM signal results in $1.2 \mathrm{~V}$ input voltage to the motor. In order to achieve a $2.4 \mathrm{kHz}$ PWM frequency, a 12-bit PWM was selected.

The noisy signal obtained from the sensor is squared up with a Schmitt Trigger. Whenever there is a pulse trigger from the sensor within a specified period of time, the microcontroller causes an interrupt and estimates the current speed. Then, it compares it with the desired one and imposes the required amount of voltage according to (9), on one of the two channels, as a PWM value.

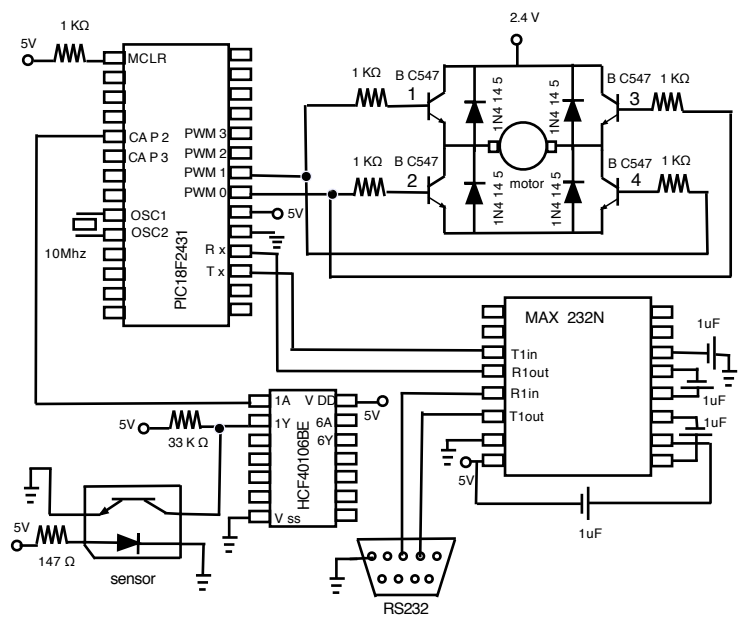

Fig. 10. Schematics for each motor control.

The presented implementation, introduces difficulties which are not encountered with regular dc motors. Due to the small size of the motor and the use of the self-made $1 \mathrm{cpr}$ encoder, the controller detects the motor signal every rising edge of the pulse to estimate the speed. Thus control is applied every 360 degrees of the rotation of the eccentric mass, and therefore, it is difficult to estimate the angle of rotation, and thus the term $m g r \sin \theta$ at different angles.

A flow chart of the control algorithm is presented in Fig. 11. At the start, all relevant registers and variables are initialized. Then, an initial PWM value is applied to both motors so that they exceed the frictional forces, and obtain an initial speed. Then, the control loop is initiated, a timer is started, and the algorithm awaits for an interrupt, when the sensor is triggered by a rising edge of the pulse. The value of the timer is stored at a special buffer, thus the period of the signal can be calculated. The microcontroller calculates the rotational speed, and estimates the error. The auxiliary control law is calculated according to:

$$
u=-K_{p} \omega+K_{I} S_{e} \Delta t
$$

where $S_{e}$ is the accumulated error, and $\Delta t=12 \mathrm{~ms}$ is the control loop time. The control voltage, $V_{s}$, is estimated, and applied as a PWM value given by:

$$
P W M=V_{s} \frac{4095}{1.2}
$$

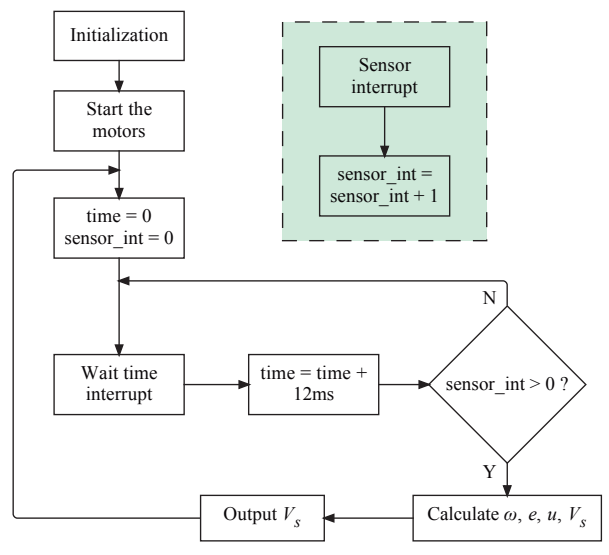

Fig. 11. Flow chart of the control algorithm for each motor.

\section{Closed-Loop ExPerimental Results}

\section{A. Micro-motor experiments}

Next, closed-loop experimental results are presented. For the experimental procedure, we applied the controller presented in (9) and (13), omitting the term containing the angle of rotation, $\theta$. This was necessary, since in practice, using the developed $1 \mathrm{cpr}$ encoder, we could not accurately estimate the angle of rotation, as explained in the previous section. By applying the control law presented in (13), and the gains obtained from the analysis in Section III, $K_{p}=49$ and $K_{I}=605$, the response of the micromotor approaches the theoretical model very well, as shown in Fig. 12. The response is acceptable even if there is a $20 \%$ error in the estimation of the parameters of the model (blue line in Fig. 12). In these plots, the desired speed is $560 \mathrm{rad} / \mathrm{s}$ and $900 \mathrm{rad} / \mathrm{s}$. The steady-state error is less than $1 \%$, and the settling time is reduced by approximately $50 \%$, in comparison to the open loop responses shown in Fig. 4.
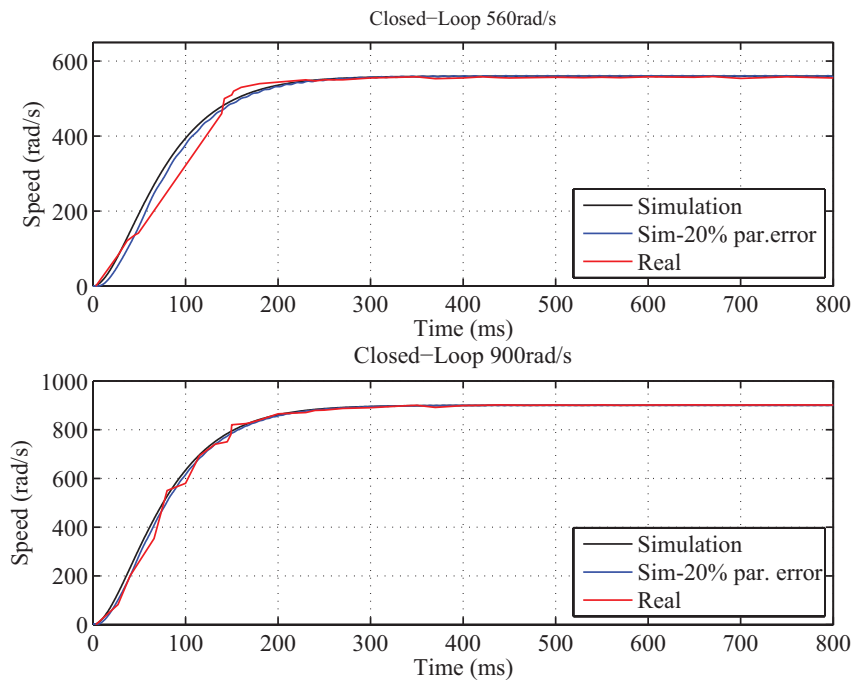

Fig. 12. The speed response at experimental closed-loop control approaches well the simulation results.

\section{B. Micro-robotic platform experiments}

As mentioned earlier, our aim is to improve the motion resolution and the bandwidth of the micro-robot, by imple- 
menting the proposed speed controller. This is especially important when two motors drive a micro-robotic platform. For example, assume that the platform is to be driven along a straight path. To minimize Cartesian errors, the two motors must have very short and equal time constants, and be able to maintain the commanded speeds constant. Any deviation in speeds during the motion will result in path deviations, which will increase with time to large position errors.

To evaluate the effect of the proposed controller on the motion of the micro-robotic platform, the micro-robotic platform was commanded to translate in a straight line. The experiment was run first using open-loop commands by applying appropriate voltages to the micromotors, and then using closed-loop commands by employing the motor speed controller given by (9) and (13). Two sets of this experiment were produced, for micro-motor desired speeds of $350 \mathrm{rad} / \mathrm{s}$ and $600 \mathrm{rad} / \mathrm{s}$. The motion of the platform was recorded by a video-microscope, and the results are presented in Fig. 13, and Fig. 14.
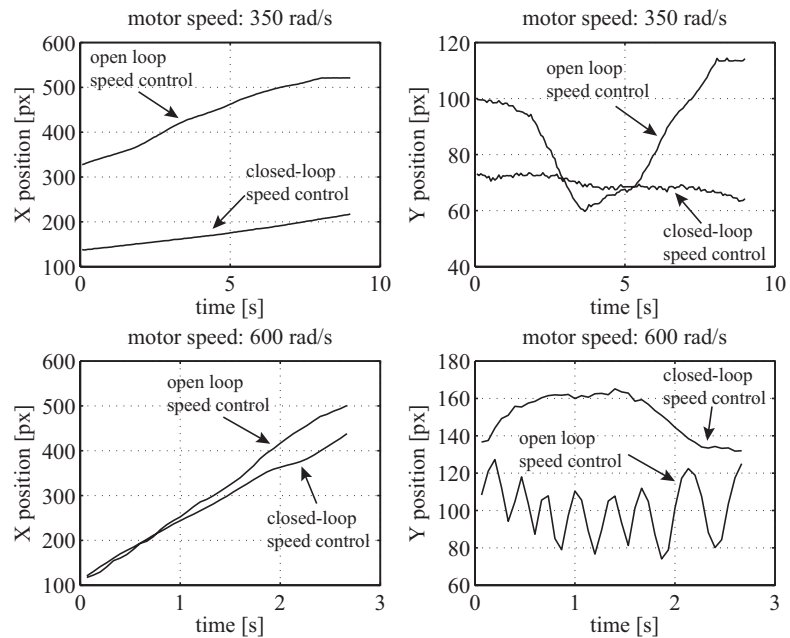

Fig. 13. The trajectory of the micro-robotic platform.

The plots on the top of Fig. 13 present the $\mathrm{X}$ and Y-axis trajectories of the micro-robotic platform, for the desired speeds of $350 \mathrm{rad} / \mathrm{s}$. The lower part of the figure shows the same trajectories for the desired speeds of $600 \mathrm{rad} / \mathrm{s}$. The Cartesian paths of the micro-robotic platform for $350 \mathrm{rad} / \mathrm{s}$ and $600 \mathrm{rad} / \mathrm{s}$ are depicted in Fig. 14.
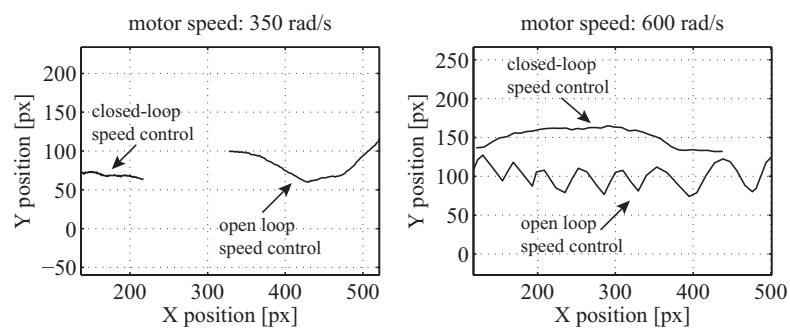

Fig. 14. The trajectory of the micro-robotic platform.

Comparing the open-loop to the closed-loop response, one can see that the use of the speed controller results in smoother and more straight trajectories, and consequently in more accurate motions, particularly in the second set, where the micro-robotic platform translates faster.

\section{CONCLUSION}

This paper presented the design and practical implementation of the speed control of a DC permanent magnet micro-motor with an eccentric mass, attached to a microrobotic platform. The dynamic model of the motor was derived, and open loop simulation and experimental results were given. A model-based closed-loop speed controller was developed, and implemented. The practical implementation of the controller was challenging, due to the motor small scale, for which no commercial incremental encoders are available. Although sensors and the means for identification were highly constrained, we presented a novel simple implementation of a 1cpr encoder, using a low-cost sensor that detects a pulse signal from the eccentric mass without requiring contact with it. Although control action is applied in every single rotation of the eccentric mass, speed control was successful as shown in the experimental results. The errors were practically eliminated, and the rotational speed response of the micro-motor was $65 \%$ faster. Moreover, experiments showed that the faster and accurate speed control at the motor level resulted in smoother platform motion with better Cartesian tracking capabilities.

In the future, we aim to improve the developed encoder, and the processing algorithm, in order to estimate the angle of rotation of the eccentric mass, and hence to reduce the ripples from the closed-loop speed signal.

\section{REFERENCES}

[1] S. Fatikow and U. Rembold, Microsystem Technology and Microrobotics, 1st ed. Springer, 2002.

[2] S. Yu and B. Nelson, "Microrobotic cell injection," in Robotics and Automation, 2001. Proceedings 2001 ICRA. IEEE International Conference on, vol. 1, may 2001, pp. 620 - 625 vol.1.

[3] P. Dario, R. Valleggi, M. C. Carrozza, M. C. Montesi, and M. Cocco, "Microactuators for microrobots: a critical survey," Journal of Micromechanics and Microengineering, vol. 2, no. 3, pp. 141-157, 1992.

[4] F. Schmoeckel and S. Fatikow, "Smart flexible microrobots for scanning electron microscope (sem) applications," Journal of Intelligent Material Systems and Structures, vol. 11, no. 3, pp. 191-198, 2000.

[5] J. Brufau et al., "Micron: Small autonomous robot for cell manipulation applications," in Robotics and Automation, 2005. ICRA 2005. Proceedings of the 2005 IEEE International Conference on, april 2005 , pp. $844-849$.

[6] S. M. Martel et al., "Flip-chip electronic system assembly process and issues for the nanowalker: a small wireless autonomous instrumented robot," in Proc. SPIE 3834, Microrobotics and Microassembly, 1999, pp. 55-62.

[7] M. Karpelson, G.-Y. Wei, and R. J. Wood, "Driving high voltage piezoelectric actuators in microrobotic applications," Sensors and Actuators A: Physical, vol. 176, no. 0, pp. 78 - 89, 2012.

[8] P. Vartholomeos and E. Papadopoulos, "Analysis, design and control of a planar micro-robot driven by two centripetal-force actuators," in Robotics and Automation, 2006. ICRA 2006. Proceedings 2006 IEEE International Conference on, may 2006, pp. 649 -654.

[9] — "Dynamics, design and simulation of a novel microrobotic platform employing vibration microactuators," Journal of Dynamic Systems, Measurement, and Control, vol. 128, no. 1, pp. 122-133, 2006.

[10] M. Rubenstein, C. Ahler, and R. Nagpal, "Kilobot: A low cost scalable robot system for collective behaviors," in Robotics and Automation (ICRA), 2012 IEEE International Conference on, may 2012, pp. $3293-3298$. 